\title{
Comparison of methods for the isolation of methicillin resistant Staphylococcus aureus
}

\author{
S Davies, P M Zadik
}

\begin{abstract}
The control of methicillin resistant Staphylococcus aureus (MRSA) relies on the rapid and sensitive detection of carriage. The roles of an enrichment broth, duration of incubation, and Baird-Parker medium containing ciprofloxacin (BPC) were evaluated in comparison with standard media in a centre where the prevalence of ciprofloxacin resistance among MRSA is over $\mathbf{9 8 \%}$. Screening swabs from 402 sites were plated onto BPC, mannitol salt agar (MSA), and MSA with methicillin (MMSA). The swabs were enriched in Tryptone-T broth with $6 \%$ salt for 24 hours and the broths subcultured onto BPC, MSA, and MMSA. MRSA was isolated from 134 swabs. Significantly more isolates were obtained by incubating culture plates for 42 hours rather than 18 hours, by the use of broth enrichment, and by addition of methicillin or ciprofloxacin to media. BPC was the most sensitive medium (107 isolates $(80 \%)$ by direct culture at 42 hours), grew the fewest contaminants, and allowed provisional reporting of $73 \%$ of isolates at 18 hours by colonial appearance and use of Staphaurex Plus rapid latex reagent. This may allow the introduction of infection control measures a day earlier than when other established methods are used.

(F Clin Pathol 1997;50:257-258)
\end{abstract}

Keywords: MRSA; methicillin resistance; ciprofloxacin resistance.

Strains of Staphylococcus aureus resistant to $\beta$-lactamase stable $\beta$-lactams (methicillin resistant $S$ aureus, MRSA) have rapidly increased in prevalence recently in England and Wales ${ }^{1}$ and are associated with significant morbidity and cost. ${ }^{2}$ Current control strategies rely on the detection of infection and colonisation and the isolation and sometimes treatment of infectious people. ${ }^{2}$ If control is to be effective then such detection should be as rapid as possible and highly sensitive. ${ }^{34}$ Ways of optimising the sensitivity of isolation have been described, involving the addition of methicillin or oxacillin to mannitol salt agar $^{4-6}$ and the use of enrichment in salt broth. ${ }^{4}$

A rapidly increasing control of infection and laboratory workload prompted us to look carefully at our methods. We decided to look at the performance of Baird-Parker medium ${ }^{7}$ (selective for $S$ aureus) containing ciprofloxacin. This initially looked promising in our area, where the predominant strain is epidemic MRSA (EMRSA) 15 which, along with some other strains we encounter, is highly resistant to ciprofloxacin. ${ }^{1}$ In 1995 and 1996 more than $98 \%$ of MRSA strains from our centre had high level resistance to ciprofloxacin. We also aimed to evaluate an enrichment procedure using Tryptone-T salt broth. Tryptone-T is a low iron content peptone which partially inhibits $S$ epidermidis but allows growth of $S$ aureus (Sheffield Public Health Laboratory, unpublished).

\section{Methods}

All screening swabs submitted for detection of MRSA during 12 working days in May and June 1996 were examined in the comparative study. Cotton tipped swabs were received in Amies transport medium and were inoculated onto half agar plates of mannitol salt agar (MSA; Unipath, Basingstoke, England) with 4 $\mathrm{mg} / 1$ methicillin (MMSA) and Baird-Parker medium (Unipath) with $8 \mathrm{mg} / 1$ ciprofloxacin (BPC). The swabs were broken off into $2 \mathrm{ml}$ of $1 \%$ Tryptone-T broth (Unipath) with $60 \mathrm{~g} / 1$ sodium chloride (TBS) which was incubated at $37^{\circ} \mathrm{C}$ for 24 hours and then subcultured onto MSA, MMSA, and BPC. All plates were incubated aerobically at $37^{\circ} \mathrm{C}$ and examined at 18 and 42 hours for typical colonies of $S$ aureus. Isolates were identified as MRSA by spot inoculation onto DNase agar (Unipath) and onto two plates of DST agar with $4 \mathrm{mg} / \mathrm{l}$ methicillin-one was incubated at $30^{\circ} \mathrm{C}$ for 18 hours and the other, containing $50 \mathrm{~g} / 1$ sodium chloride, at $37^{\circ} \mathrm{C}$ for 18 hours and examined for visible growth. Strains were classified as being EMRSA 15 or EMRSA 16 by their urease reaction on Christensen's urea slope and by their resistance pattern to erythromycin, gentamicin, ciprofloxacin, and trimethoprim, ${ }^{1}$ or they were phage typed at the Staphylococcal Reference Unit, PHLS Central Public Health Laboratory, Colindale. All suspected MRSA colonies from BPC and MMSA were tested by Staphaurex Plus rapid latex reagent (Murex, Dartford, England).

Analysis of comparison of isolation rates was done by the two paired proportion method $^{8}$ in which the numbers of specimens positive solely by one test were compared with the numbers positive solely by the other test.

\section{Results}

Four hundred and two swabs were taken from 98 patients. Of these 85 were nose swabs, 93 from groin or perineum, and 71 from the axillae. MRSA was detected from 134 swabs by at least one method. These strains came from 50 
Table 1 Number of isolates detected and sensitivity of detection from 402 specimens of which 134 yielded MRSA

\begin{tabular}{|c|c|c|c|c|}
\hline & \multicolumn{2}{|c|}{ Direct culture } & \multicolumn{2}{|c|}{ Broth subculture } \\
\hline & $\begin{array}{l}\text { Isolates } \\
(n)\end{array}$ & $\begin{array}{l}\text { Sensitivity } \\
(\%)\end{array}$ & $\begin{array}{l}\text { Isolates } \\
\text { (n) }\end{array}$ & $\begin{array}{l}\text { Sensitivity } \\
(\%)\end{array}$ \\
\hline \multicolumn{5}{|c|}{ Mannitol salt medium } \\
\hline $18 \mathrm{~h}$ & 77 & 58 & 96 & 72 \\
\hline $42 \mathrm{~h}$ & 86 & 64 & 110 & 82 \\
\hline \multicolumn{5}{|c|}{ Mannitol salt medium + methicillin } \\
\hline $18 \mathrm{~h}$ & 89 & 66 & 113 & 84 \\
\hline $42 \mathrm{~h}$ & 89 & 73 & 118 & 88 \\
\hline \multicolumn{5}{|c|}{ Baird-Parker medium + ciprofloxacin } \\
\hline $18 \mathrm{~h}$ & 98 & 73 & 115 & 86 \\
\hline $42 \mathrm{~h}$ & 107 & 80 & 122 & 91 \\
\hline
\end{tabular}

Table 2 Significance of differences in performance ( $p$ values) of media for isolation of $M R S A$ after 42 incubation

\begin{tabular}{lcllll}
\hline MSA-direct & \multicolumn{5}{l}{} \\
0.036 & MMSA-direct & & \\
$<0.001$ & 0.061 & BPC-direct & \\
$<0.001$ & 0.058 & 0.74 & MSA-subculture & \\
$<0.001$ & $<0.001$ & 0.063 & 0.077 & MMSA-subculture \\
$<0.001$ & $<0.001$ & 0.008 & 0.023 & 0.39 & BPC-subculture \\
\hline
\end{tabular}

Table 3 Numbers of non-MRSA isolates investigated

\begin{tabular}{lcc}
\hline & Direct culture $(n)$ & Broth subculture $(n)$ \\
\hline Mannitol salt medium & 86 & 128 \\
$18 \mathrm{~h}$ & 119 & 130 \\
$42 \mathrm{~h}$ & 29 & 59 \\
Mannitol salt medium + methicillin & 63 & 75 \\
$18 \mathrm{~h}$ & 5 & 10 \\
$42 \mathrm{~h}$ & 6 & 12 \\
Baird-Parker medium + ciprofloxacin & & \\
$18 \mathrm{~h}$ & & \\
$42 \mathrm{~h}$ & & \\
\hline
\end{tabular}

patients, 49 of whom were inpatients at the Northern General Hospital. Forty six patients carried EMRSA 15, three carried EMRSA 16, and a further patient carried a ciprofloxacin sensitive strain of an unrelated phage type. Table 1 shows the performance of the different media. Sensitivity was increased by between $11 \%$ and $18 \%$ using broth subculture, by $7 \%$ when incubation time of direct cultures was increased from 18 to 42 hours, by $9 \%$ when methicillin was added to MSA, and by $16 \%$ when BPC was compared to MSA for direct culture. Table 2 shows the significance of the differences between the various isolation rates after 42 hours of incubation.

Colonies of MRSA gave a visible lipase reaction on BPC, and all strains isolated using BPC gave a positive reaction with Staphaurex Plus, allowing the presumptive reporting of ciprofloxacin resistant $S$ aureus. It was not possible to test isolates from MMSA by Staphaurex Plus reagent because of autoagglutination when emulsification was attempted.

Table 3 shows the number of colonies picked off each medium that proved not to be MRSA. These were predominantly coagulase negative staphylococci, but methicillin sensitive $S$ aureus were also frequently cultured from MSA. Methicillin sensitive, ciprofloxacin resistant $S$ aureus was detected from three specimens on BPC by direct culture and from five after enrichment. These were all from one patient.

\section{Discussion}

The results confirm useful improvements in sensitivity by the use of agar media containing selective antimicrobials ${ }^{4-6}$ and by enrichment in salt broth. ${ }^{4}$ They also show a clear advantage in incubating the plates for up to 42 hours.

The study did not involve any comparison between salt broths and did not point to better performance than with Oxoid No 2 nutrient broth with $8 \%$ salt. ${ }^{4}$ Clearly, the use of an enrichment broth could be justified by the greater yield of positives but cannot replace direct culture because of a delay in results.

BPC was more sensitive than MMSA for direct culture in this study. Any single centre study will deal with a limited spectrum of strains and our results may not apply to other centres and certainly will not apply where strains sensitive to ciprofloxacin are common. $\mathrm{BPC}$ has the other great advantage of allowing us to issue a presumptive positive report at 18 hours (one day earlier than with MMSA) and since we see very few strains of $S$ aureus sensitive to methicillin and resistant to ciprofloxacin, the specificity is also high. MMSA grew significantly more non-MRSA strains requiring investigation than did BPC. Colonies from MMSA could not be emulsified as has been previously reported using a similar medium, ${ }^{5}$ preventing confirmation of identity by rapid latex reagent.

Although it may appear unconventional to screen for strains with one property (methicillin resistance) by means of another property (ciprofloxacin resistance), BPC performed better than other media even in this trial of unselected patients. In practice there would be even less difficulty since when we screen a patient or a ward we usually know the susceptibility of the strain we are looking for, as it has been detected using non-selective media in the course of routine bacteriological examination. When we are looking for ciprofloxacin sensitive strains we would obviously not use BPC. Where the nature of the strain is unknown the use of BPC for direct culture with MMSA after broth enrichment would be the combination that would give the best balance of speed and sensitivity, although at increased cost and expenditure of time. The cost of this additional step could be reduced by processing several swabs from one person in one broth. ${ }^{4}$

1 CDSC. Epidemic methicillin-resistant Staphylococcus aureus. Commun Dis Rep 1995;5:165.

2 Working party report. Revised guidelines for the control of epidemic methicillin-resistant Staphylococcus aureus. $\mathcal{f}$ Hosp Infect 1990;16:351-77.

3 Kristinsson KG, Fenton P, Norman P. Control of epidemic methicillin-resistant Staphylococcus aureus [letter]. Lance 1987;i:274-5.

4 Cookson BD, Webster M, Phillips I. Control of epidemic methicillin-resistant Staphylococcus aureus [letter]. Lancet 1987;i:696.

5 van Enk RA, Thompson DT. Use of a primary isolation medium for recovery of methicillin-resistant Staphylococcus aureus. $\mathcal{F}$ Clin Microbiol 1992;30:504-5.

6 Lally RT, Ederer MN, Woolfrey BF. Evaluation of mannito salt agar with oxacillin as a screening medium for methicillin-resistant Staphylococcus aureus. $f$ Clin Microbiol 1985;22:501-4.

7 Baird-Parker AC. An improved diagnostic and selective medium for isolating coagulase positive staphylococci. $\mathcal{F}$ Appl Bacteriol 1962;25:12-19.

8 Altman DG. Practical statistics for medical research. London: Chapman and Hall, 1991:235-9. 